\title{
Controlled spontaneous emission in plasmonic whispering gallery antennas
}

\author{
Ernst Jan R. Vesseur ${ }^{a)}$ and Albert Polman \\ Center for Nanophotonics, FOM Institute AMOLF, Science Park 104, 1098 XG Amsterdam, The Netherlands
}

(Received 13 October 2011; accepted 14 November 2011; published online 9 December 2011)

\begin{abstract}
We present a plasmonic whispering gallery nanoantenna doped with an ATTO680 dye that shows cavity-modified spontaneous emission. The plasmonic ring antenna consists of a circular groove cavity in a single-crystal Au surface that sustains resonances with different azimuthal and radial mode order. We observe spectral reshaping of the dye emission that can be tuned over a broad band by varying the cavity resonance conditions. (c) 2011 American Institute of Physics. [doi:10.1063/1.3665622]
\end{abstract}

Optical nanoantennas can be used to control the coupling of an emitter to the far field, thereby tuning the angular distribution $^{1-4}$ and polarization ${ }^{5,6}$ of the emission. As a result of the emitter-antenna coupling, the spontaneous emission spectrum and decay rate of the emitter can be strongly modified, ${ }^{1,7-9}$ and consequently, its emission spectrum is reshaped dependent on the antenna resonances. The coupling of an antenna to an emitter is strong when the emitter is positioned at the region in which the resonant field of the antenna is high. Nanoantennas often consist of single metal particles, such as spheres ${ }^{8,10}$ or rods, ${ }^{11,12}$ but they can also consist of dimer geometries ${ }^{13-17}$ or particle arrays. ${ }^{18-21}$ In these antennas, the field is maximum in very small hotspots. Consequently, maximizing the coupling of an emitter to these antennas relies on careful positioning of the emitter into these high-field hotspots.

Here, we introduce a ring cavity in a planar Au surface, consisting of a circular V-groove, as an optical nanoantenna geometry that allows for coupling of emitters to the cavity resonances over a large cavity volume inside the groove. The circular groove sustains surface plasmon resonances that are due to groove-bound surface plasmon polaritons that are confined between the two closely spaced groove sidewalls. ${ }^{22}$ Resonances occur when the circumference of the ring equals an integer number $m$ of plasmon wavelengths. The lowestorder azimuthal resonance in these plasmonic ring cavities occurs if the ring circumference equals a single plasmon wavelength $\lambda_{p}$. As we have shown previously, ${ }^{23}$ these plasmon ring cavities also support many high-order azimuthal modes for increasing diameter. In addition, for deeper grooves, higher-order radial modes (indicated by $n$ ) are observed that correspond to an increase in the number of antinodes in the radial direction of the cavity.

The resonant field in the V-groove is "squeezed" between the sidewalls, ${ }^{24}$ analogous to the plasmon in a metal-insulator-metal geometry. ${ }^{25}$ Despite the tight confinement of the groove plasmon, the V-grooves are open at the top and allow the cavity modes to be accessed from the outside. Emitters can, thus, be incorporated into the high-field region, making this cavity an ideal geometry to study the interaction between emitters and antenna resonances. In this paper, we show how the fluorescence of dye molecules

$\overline{{ }^{a} \text { Electronic mail: vesseur@amolf.nl. }}$ inside the groove of the plasmonic ring antenna is strongly affected by the tunable cavity resonances.

Arrays of plasmonic ring antennas were fabricated using focused-ion-beam (FIB) milling. The antennas consist of a circular groove milled into a smooth Au surface. A representative structure and its cross-section are shown in Figure 1. The V-shaped grooves are approximately $100 \mathrm{~nm}$ wide at the top with a depth $d$ ranging from 25 to $500 \mathrm{~nm}$. Ring radii $r$ are in the range $100-300 \mathrm{~nm}$. The substrate, consisting of large $(\sim 10 \mu \mathrm{m})$ crystal domains, was obtained by thermal evaporation and template stripping off a mica substrate. Arrays composed of multiple rings are made within a single crystal domain allowing for smooth FIB milling that does not suffer from differences in milling rate between crystal grains. A rectangular array of $20 \times 17$ rings with a $2 \mu \mathrm{m}$ pitch was milled. At each column, the depth is increased; for each row, the radius is increased.

Ring cavities were coated by a polymer layer that was doped with ATTO680 dye molecules. The layer was obtained by spin coating a solution of $3 \times 10^{-5} \mathrm{M}$ dye and 5 wt. \% poly-vinyl butyral (PVB) in ethyl-lactate onto the Au substrate. ${ }^{7}$ The resulting layer thickness is $10-50 \mathrm{~nm}$ as can be seen in Fig. 1(b). The bottom half of the groove void of the plasmon cavities is infilled with PVB with the exception of small air inclusions at the bottom.

White-light scattering measurements were taken using a $60 \times \mathrm{NA}=0.9$ objective which focused white light from an incandescent lamp source onto the sample surface. Scattered light was collected by the same objective and, using a beam splitter, projected onto a $9 \mu \mathrm{m}$ core diameter fiber guiding the light into a spectrometer equipped with a thermo-
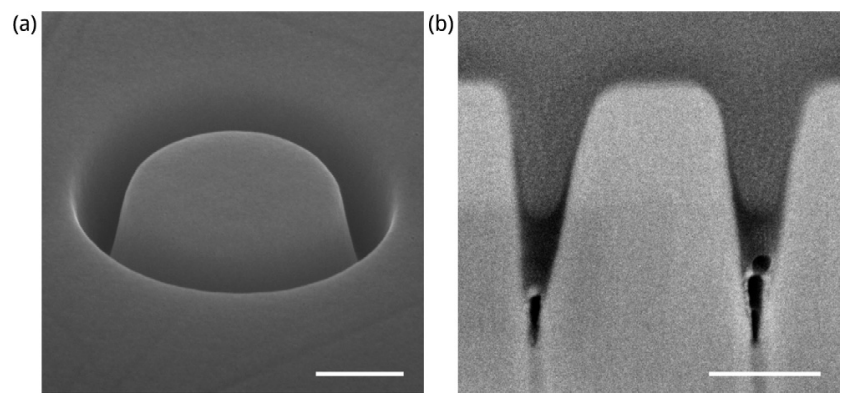

FIG. 1. Scanning electron micrographs of plasmonic ring antenna with radius $r=300 \mathrm{~nm}$ and groove depth $d=500 \mathrm{~nm}$. (b) FIB-milled cross-section through a ring antenna infilled with a dye-doped PVB polymer layer. Scale bars: $200 \mathrm{~nm}$. 
electrically cooled CCD array detector. Spectra were divided by the scattering spectrum of unstructured $\mathrm{Au}$ to obtain the relative reflection.

Scanning confocal optical microscopy was performed using the same setup. A $5 \mathrm{~mW}$ diode laser emitting at $640 \mathrm{~nm}$ was used as an excitation source and filtered through a $650 \mathrm{~nm}$ shortpass filter; in the detection path, a $700 \mathrm{~nm}$ longpass filter was used.

As an additional method to probe the antenna resonances, we measured cathodoluminescence (CL) spectra of the ring cavities. This technique involves the excitation of a single ring cavity using an electron beam. The spectrum that is subsequently emitted carries the signature of the cavity resonances. The signal is corrected for system response (more details about CL spectroscopy are described elsewhere ${ }^{23}$ ).

Before studying the interaction between the antenna resonances and the dye molecules, we probe the ring cavities using white light scattering and CL spectroscopy.

Figure 2 shows white-light scattering and CL emission spectra of a representative $r=225 \mathrm{~nm}, d=50 \mathrm{~nm}$ ring. Before infilling, the ring resonance is at $700 \mathrm{~nm}$, as can be seen in the CL spectrum (black). The scattering spectrum (red) shows a dip around this wavelength due to coupling of the incident light to the $m=1$ resonance. The scattering spectrum results from the interference between scattered light from the antenna and light that is directly reflected from the planar sample surface, giving rise to a Fano lineshape. ${ }^{4}$ Since the CL spectrum is not affected by such interference effects, there is an offset between the minimum in the scattering spectrum and the maximum in the CL spectrum.

As a result of infilling the groove void with a polymer layer, the resonances undergo a red shift. ${ }^{26}$ After infilling, the scattering measurement shows a dip at $750 \mathrm{~nm}$ (blue curve), showing that the resonance has indeed shifted to a longer wavelength. A change in the shape of the scattering spectrum is also observed upon infilling, which is attributed to a change in the interference conditions due to the presence

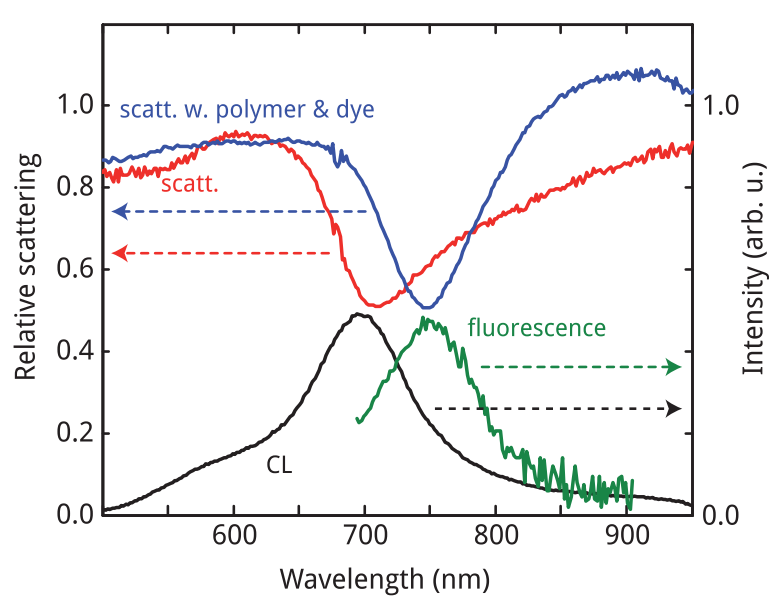

FIG. 2. (Color) Resonance spectra of a single ring antenna $(r=225 \mathrm{~nm}$, $d=50 \mathrm{~nm}$ ) before and after infilling with dye-containing polymer. Black curve: cathodoluminescence spectrum, showing a clear resonant peak. Red curve: relative white-light reflection spectrum showing a Fano line shape at the resonance wavelength. As a result of a polymer layer covering the antenna, the white-light reflection spectrum red shifts (blue curve). Green curve: fluorescence enhancement of dye molecules incorporated in the polymer layer. of the PVB layer on the Au surface. The observed red shift is smaller than what would be expected assuming full infiltration of the V-groove with the polymer $\left(n_{\text {polymer }}=1.49\right)$. We attribute this to the presence of air voids at the bottom of the groove, which effectively lower the index, and the fact that a significant fraction of the resonant field extends above the polymer film.

The ATTO680 fluorescent dye used in these experiments has a quantum efficiency of $\sim 30 \%$ with a broad luminescence above $700 \mathrm{~nm}$ and a maximum absorption at $680 \mathrm{~nm}$. Figure 3 shows the emission spectrum (labeled "reference") of ATTO680 molecules embedded in a 10-nm layer of PVB on a flat Au substrate. The spectrum recorded from ATTO680 dye molecules inside the $r=225 \mathrm{~nm}$, $d=50 \mathrm{~nm}$ ring cavity (same as Fig. 2) is also shown in Fig. 3. This spectrum is clearly different from the reference and shows an enhancement of the fluorescence in the central part of the spectrum. The fluorescence enhancement spectrum, obtained by the division of the cavity spectrum by the reference spectrum, is also plotted in Fig. 3. We observe an enhancement of the fluorescence over a broad range around $750 \mathrm{~nm}$. The fluorescence enhancement at $750 \mathrm{~nm}$ is more than 5 times higher than at the edges of the spectrum, suggesting an enhancement of the radiative rate at this wavelength that is at least a factor 5. The fluorescence enhancement spectrum is also plotted in Fig. 2. Clearly, the fluorescence is enhanced right at the cavity resonance.

Taking advantage of the large tunability of plasmon cavity resonance, we now make a systematic comparison of the fluorescence enhancement for different cavity geometries. Figure 4 shows a confocal microscopy scan of an array of cavities with different depth and diameter. In the confocal scan, the fluorescence spectrum was recorded for every pixel in a rectangular grid. In this figure, only the emission at $710 \mathrm{~nm}$ and $800 \mathrm{~nm}$ is shown (spectral bandwidth $10 \mathrm{~nm}$ ). Each bright dot in the figure corresponds to an individual ring cavity. Data are normalized to the fluorescence from a planar part of the substrate. The cavities have a diameter that increases from left to right; the groove depth increases from bottom to top. Clearly, the fluorescence enhancement

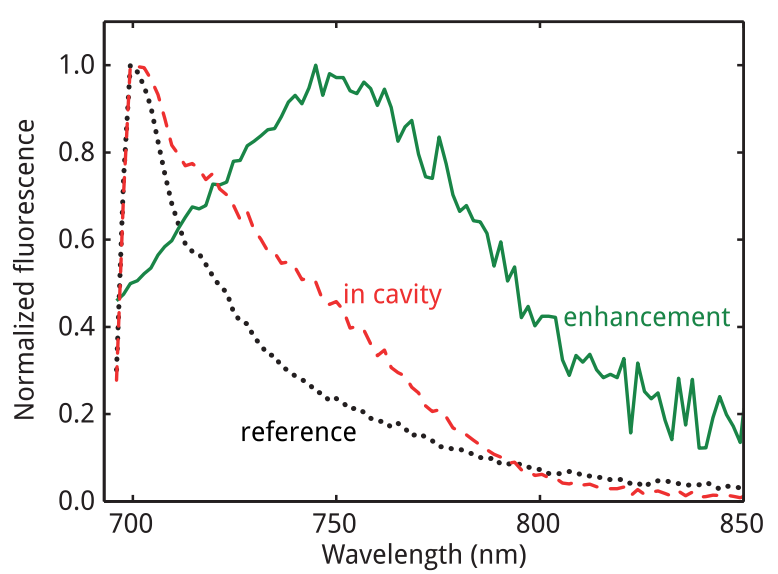

FIG. 3. (Color) Normalized fluorescence spectrum of ATTO 680 dye embedded in PVB inside a $r=225 \mathrm{~nm}, d=50 \mathrm{~nm}$ plasmon ring cavity (red). A reference spectrum from dye in a $10 \mathrm{~nm}$ polymer layer on unstructured $\mathrm{Au}$ is also shown (black). The fluorescence enhancement in the ring cavity is shown by the green curve $\left(\lambda_{\text {pump }}=640 \mathrm{~nm}\right)$. 

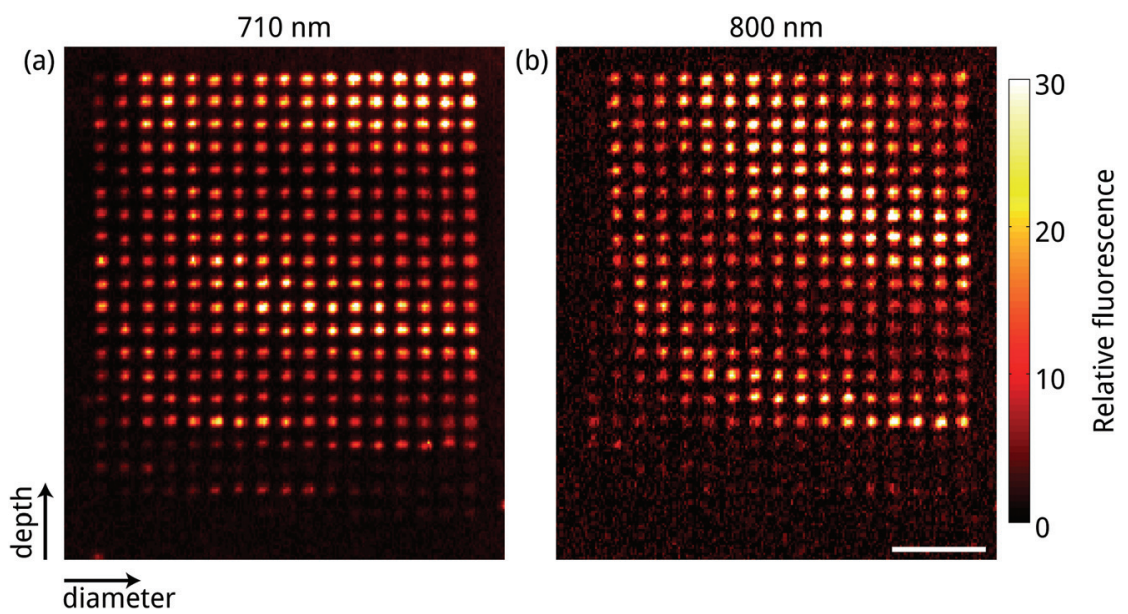

FIG. 4. (Color) Confocal microscopy scan of ATTO680 fluorescence in an array of $17 \times 20$ ring cavities. The rings have groove depths ranging from $25 \mathrm{~nm}$ (bottom) to $500 \mathrm{~nm}$ (top) and radii of $100 \mathrm{~nm}$ (left)-300 nm (right). Excitation was at $640 \mathrm{~nm}$. Plotted in these scans is the fluorescence in a $10-\mathrm{nm}$ band around (a) $710 \mathrm{~nm}$ and (b) $800 \mathrm{~nm}$. Data are normalized to fluorescence of the planar part of the Au substrate. Scale bar: $10 \mu \mathrm{m}$. strongly depends on cavity geometry. The bright cavities appear as bands in the image. This is due to the fact that different combinations of depth and diameter yield the same resonant wavelength. The figure clearly shows that the fluorescence is enhanced in ring cavities over a broad range of cavity sizes and shapes, due to their cavity resonance. These bands are both observed at 710 and $800 \mathrm{~nm}$ but at different cavity geometries. Exploiting the tunability of the ring resonances, the fluorescence enhancement can be engineered throughout the entire emission band of the ATTO680 molecules. Comparison of the data in Fig. 4 with white-light scattering data (not shown) shows that the bright fluorescence bands correspond to resonances, with $m=1$ and $n=1,2,3$., that all have a dipolar character. Most cavities in the array have depth $d>100 \mathrm{~nm}$. In these deep structures, the resonance spectrum is dominated by several higher-order $n$-modes. These modes efficiently couple to free-space light.

Finally, we note that resonance effects near the 640-nm laser excitation wavelength do not play a role in the intensity enhancements observed.

In conclusion, we have demonstrated that plasmonic ring cavities can be used to reshape the emission of dye molecules over a broad spectral band. Due to its large mode volume, a single plasmonic ring cavity causes enhanced spontaneous emission for a large number of dye molecules. Due to the tunability of the cavity resonances, the antenna's emission pattern is highly tunable. Moreover, the directional emission can be strongly controlled. ${ }^{4}$ When infiltrated with a high-gain medium, these cavities may also serve as extremely small plasmon lasers.

This work is a part of the research program "Microscopy and modification of nanostructures with focused electron and ion beams" (MMN) of the "Stichting voor Fundamenteel Onderzoek der Materie" (FOM), which is financially supported by the "Nederlandse organisatie voor Wetenschappelijk Onderzoek" (NWO). The MMN program is co-financed by FEI Company. The authors acknowledge Yichen Zhang, Jaime Gómez-Rivas, and F. Javier García de Abajo for discussions.
${ }^{1}$ G. Vecchi, V. Giannini, and J. G. Rivas, Phys. Rev. Lett. 102, 146807 (2009).

${ }^{2}$ T. Kosako, Y. Kadoya, and H. F. Hofmann, Nature Photon. 4, 312 (2010).

${ }^{3}$ A. G. Curto, G. Volpe, T. H. Taminiau, M. P. Kreuzer, R. Quidant, and N. F. van Hulst, Science 329, 930 (2010).

${ }^{4}$ E. J. R. Vesseur and A. Polman, "Plasmonic Whispering Gallery Cavitite as Optical Nanoantennas," Nano Lett. (in press), DOI: 10.1021/nl203418c.

${ }^{5}$ T. H. Taminiau, F. D. Stefani, F. B. Segerink, and N. F. van Hulst, Nature Photon. 2, 234 (2008).

${ }^{6}$ H. Mertens, J. S. Biteen, H. A. Atwater, and A. Polman, Nano Lett. 6, 2622 (2006).

${ }^{7}$ O. Muskens, V. Giannini, J. Sánchez-Gil, and J. Gómez-Rivas, Nano Lett. 7, 2871 (2007).

${ }^{8}$ M. Ringler, A. Schwemer, M. Wunderlich, A. Nichtl, K. Kurzinger, T. A. Klar, and J. Feldmann, Phys. Rev. Lett. 100, 203002 (2008).

${ }^{9}$ A. Kinkhabwala, Z. Yu, S. Fan, Y. Avlasevich, K. Müllen, and W. E. Moerner, Nature Photon. 3, 654 (2009).

${ }^{10}$ H. Mertens, "Controlling plasmon-enhanced luminescence," Ph.D. thesis, FOM Institute for Atomic and Molecular Physics (AMOLF), Utrecht University, 2007.

${ }^{11}$ J. Krenn, G. Schider, W. Rechberger, B. Lamprecht, A. Leitner, and F. Aussenegg, Appl. Phys. Lett. 77, 3379 (2000).

${ }^{12}$ L. Novotny, Phys. Rev. Lett. 98, 266802 (2007).

${ }^{13}$ P. Mühlschlegel, H. J. Eisler, O. J. F. Martin, B. Hecht, and D. W. Pohl, Science 308, 1607 (2005).

${ }^{14}$ O. L. Muskens, V. Giannini, J. A. Sánchez-Gil, and J. G. Rivas, Opt. Express 15, 17736 (2007).

${ }^{15}$ P. Ghenuche, S. Cherukulappurath, T. H. Taminiau, N. F. van Hulst, and R. Quidant, Phys. Rev. Lett. 101, 116805 (2008).

${ }^{16}$ M.-W. Chu, V. Myroshnychenko, C. H. Chen, J.-P. Deng, C.-Y. Mou, and F. J. García de Abajo, Nano Lett. 9, 399 (2009).

${ }^{17}$ J.-S. Huang, J. Kern, P. Geisler, P. Weinmann, M. Kamp, A. Forchel, P. Biagioni, and B. Hecht, Nano Lett. 10, 2105 (2010).

${ }^{18}$ S. A. Maier, P. G. Kik, and H. A. Atwater, Appl. Phys. Lett. 81, 1714 (2002).

${ }^{19}$ L. A. Sweatlock, S. A. Maier, H. A. Atwater, J. J. Penninkhof, and A. Polman, Phys. Rev. B 71, 235408 (2005).

${ }^{20}$ R. de Waele, A. F. Koenderink, and A. Polman, Nano Lett. 7, 2004 (2007).

${ }^{21}$ S. Bidault, F. J. García de Abajo, and A. Polman, J. Am. Chem. Soc. 130, 2750 (2008)

${ }^{22}$ E. J. R. Vesseur, F. J. García de Abajo, and A. Polman, Nano Lett. 9, 3147 (2009).

${ }^{23}$ E. J. R. Vesseur, R. de Waele, M. Kuttge, and A. Polman, Nano Lett. 7, 2843 (2007).

${ }^{24}$ S. I. Bozhevolnyi, V. S. Volkov, E. Devaux, and T. W. Ebbesen, Phys. Rev. Lett. 95, 046802 (2005).

${ }^{25}$ J. A. Dionne, L. A. Sweatlock, H. A. Atwater, and A. Polman, Phys. Rev. B 72, 075405 (2005).

${ }^{26}$ K. C. Vernon, D. K. Gramotnev, and D. F. P. Pile, J. Appl. Phys. 103, 034304 (2008). 